\title{
A survey on the spectral theory of nonnegative tensors
}

\author{
Kungching Chang ${ }^{1, *,}$, Liqun $\mathrm{Qi}^{2}$ and Tan Zhang ${ }^{3}$ \\ ${ }^{1}$ LMAM, School of Mathematical Sciences, Peking University, Beijing 100871, China \\ ${ }^{2}$ Department of Applied Mathematics, The Hong Kong Polytechnic University, Hung Hom, Kowloon, Hong Kong \\ ${ }^{3}$ Department of Mathematics and Statistics, Murray State University, Murray, KY 42071, USA
}

\begin{abstract}
SUMMARY
This is a survey paper on the recent development of the spectral theory of nonnegative tensors and its applications. After a brief review of the basic definitions on tensors, the $H$-eigenvalue problem and the $Z$-eigenvalue problem for tensors are studied separately. To the $H$-eigenvalue problem for nonnegative tensors, the whole Perron-Frobenius theory for nonnegative matrices is completely extended, while to the $Z$-eigenvalue problem, there are many distinctions and are studied carefully in details. Numerical methods are also discussed. Three kinds of applications are studied: higher order Markov chains, spectral theory of hypergraphs, and the quantum entanglement. Copyright (c) 2013 John Wiley \& Sons, Ltd.
\end{abstract}

Received 12 April 2012; Revised 5 August 2013; Accepted 18 August 2013

KEY WORDS: $\quad$ eigenvalues for tensors; Perron-Frobenius theorem; high order Markov chain

\section{INTRODUCTION}

In 2005, independently, Lim [1] and Qi [2] introduced eigenvalues for higher order tensors. Since then, spectral theory of tensors developed rapidly, find applications or links with automatical control [2], spectral hypergraph theory [3-13], higher order Markov chains [14-16], magnetic resonance imaging [17,18], algebraic geometry [19,20], Finsler geometry [21], quantum entanglement $[22,23]$, image authenticity verification [24], and so on.

However, it was discovered that for general higher order tensor eigenvalue problem, even the order is low, say three or four, the problem is NP-hard [25]. This poses a big difficulty for the development of spectral theory of tensors. On the hand, it was also found, for some special classes of tensors, the eigenvalue problem is computable, and relative theory and algorithms for matrices with such special structures can be generalized to higher order tensors without difficulty. Notably, for nonnegative tensors, that is, tensors with nonnegative entries, the whole Perron-Frobenius theory for nonnegative matrices can be extended. Eigenvalues of nonnegative tensors have also applications in higher order Markov chains, spectral hypergraph theory, and the quantum entanglement. Thus, this attracts researchers to study the theory, algorithms and applications of eigenvalues of nonnegative tensors. Many papers appeared in this area. This paper aims to survey the progress in this area, the theory, algorithms and applications of eigenvalues of nonnegative tensors.

The remainder of this paper is distributed as follows. In Section 2, we review the general spectral theory of nonnegative tensors. We then review the $H$-spectral theory and the $Z$-spectral theory of nonnegative tensors in Sections 3 and 4, respectively. In Sections 5-7, we review three applications of eigenvalues of nonnegative tensors: higher order Markov chains, spectral hypergraph theory, and the quantum entanglement.

\footnotetext{
*Correspondence to: Kungching Chang, LMAM, School of Mathematical Sciences, Peking University, Beijing 100871, China.

${ }^{\dagger}$ E-mail: kcchang@math.pku.edu.cn
} 
The spectral theory for nonnegative matrices has had a profound impact on both theoretical and applicable mathematics. The centerpiece of this theory resides on the classical Perron-Frobenius theorems as well as some of their important consequences. In this expository article, we intend to give a brief survey to incorporate some of the most recent developments of the various spectral theories for nonnegative tensors along these lines. There is a large volume of published and unpublished work in this field; we apologize in advance if we fail to cite the work of some of our peers as any such oversight is unintentional.

\section{SPECTRAL THEORY OF NONNEGATIVE MATRICES}

A second-order $n$-dimensional real (or complex) tensor $A$ is the $n \times n$ real (or complex) matrix $A=\left(a_{i j}\right)$. It can also be viewed as a linear endomorphism on $\mathbb{R}^{n}$ (or $\mathbb{C}^{n}$ ); hence, the eigenvalue problem for $A$ is a linear problem. In particular, the spectral radius $r(A)$ of $A$ is defined to be

$$
r(A)=\max \{|\lambda| \mid \lambda \text { is a real or complex eigenvalue of } A\} .
$$

According to Gelfand's formula,

$$
r(A)=\lim _{n \rightarrow \infty}\left\|A^{n}\right\|^{\frac{1}{n}},
$$

where $\|\cdot\|$ denotes the operator norm. Thus, $r(A)$ is an intrinsic property of $A$ as it is entirely determined by $A$ itself.

An $m$-order $n$-dimensional real tensor $\mathcal{A}$ consists of $n^{m}$ entries in $\mathbb{R}$ :

$$
\mathcal{A}=\left(a_{i_{1} \cdots i_{m}}\right), \quad a_{i_{1} \cdots i_{m}} \in \mathbb{R}, \quad 1 \leqslant i_{1}, \ldots, i_{m} \leqslant n .
$$

However, for $m>2$, the resulting eigenvalue problem is no longer a linear problem due to the different algebraic structures of the associated eigenvalue problems induced by $\mathcal{A}$ as we will discuss in the subsequent sections. We shall denote the set of all $m$-order $n$-dimensional tensors by $\mathbb{R}^{[m, n]}$ and the set of all nonnegative $m$-order $n$-dimensional tensors by $\mathbb{R}_{+}^{[m, n]}$ henceforth.

For convenience, we recall the following fundamental theorems in the spectral theory of nonnegative matrices. One may find detailed treatment of these results in the standard textbooks $[26,27]$.

The Perron-Frobenius theorem, which lays the foundation for the spectral theory of nonnegative matrices, has the following two forms.

Theorem 2.1

(Perron-Frobenius theorem, Weak Form) If $A$ is a nonnegative square matrix, then

1. $r(A)$, the spectral radius of $A$, is an eigenvalue.

2. There exists a nonnegative vector $x_{0} \neq 0$ such that

$$
A x_{0}=r(A) x_{0} .
$$

Definition 2.2. A square matrix $A$ is said to be reducible if it can be placed into block uppertriangular form by simultaneous row/column permutations. A square matrix that is not reducible is said to be irreducible.

\section{Theorem 2.3}

(Perron-Frobenius theorem, Strong Form) If $A$ is an irreducible nonnegative square matrix, then

1. $r(A)>0$ is an eigenvalue.

2. There exists a positive vector $x_{0}>0$, that is, all components of $x_{0}$ are positive, such that $A x_{0}=r(A) x_{0}$.

3. (Uniqueness) If $\lambda$ is an eigenvalue with a nonnegative eigenvector, then $\lambda=r(A)$. 
4. $r(A)$ is a simple eigenvalue of $A$.

5. If $\lambda$ is an eigenvalue of $A$, then $|\lambda| \leqslant r(A)$.

Concerning the distribution of eigenvalues on the spectral circle

$$
\{\lambda \in \mathbb{C}|| \lambda \mid=r(A)\},
$$

we have the following.

\section{Theorem 2.4}

Let $A$ be an irreducible nonnegative matrix. If $A$ has $k$ distinct eigenvalues of modulus $r(A)$, then the eigenvalues are $r(A) e^{i 2 \pi j / k}$, where $j=0,1, \cdots, k-1$.

We call the number $k$ the cyclic index of $A$.

The special subset of nonnegative irreducible matrices known as primitive matrices plays an important role in the calculation of the spectral radius of $A$. We denote $\mathbb{R}_{+}^{n}=\left\{\left(x_{1}, \ldots, x_{n}\right) \in\right.$ $\left.\mathbb{R}^{n} \mid x_{i} \geqslant 0,1 \leqslant i \leqslant n\right\}$, the positive cone in $\mathbb{R}^{n}$ and $\mathbb{R}_{++}^{n}$ its interior. We recall:

Definition 2.5. An irreducible nonnegative matrix $A$ is said to be primitive if the only nonempty subset of the boundary of $\mathbb{R}_{+}^{n}$, which is invariant under the action of $A$ is $\{\mathbf{0}\}$.

There are many equivalent characterizations of primitivity for matrices, one of which is the following.

\section{Theorem 2.6}

$A$ is a primitive matrix if and only if $A$ has cyclic index 1 .

There is also a minimax characterization of the spectral radius for irreducible nonnegative matrices due to Collatz and Wielandt. Namely,

\section{Theorem 2.7}

(Collatz-Wielandt) Assume $A$ is an irreducible nonnegative $n \times n$ matrix, then

$$
\min _{x \in \mathbb{R}_{++}^{n}} \max _{\left\{i \mid x_{i}>0\right\}} \frac{(A x)_{i}}{x_{i}}=r(A)=\max _{x \in \mathbb{R}_{++}^{n}} \min _{\left\{i \mid x_{i}>0\right\}} \frac{(A x)_{i}}{x_{i}} .
$$

This characterization of $r(A)$ can be numerically implemented via the power method:

Let $A \geqslant 0$ be an $n \times n$ irreducible matrix and let $y^{0} \in \mathbb{R}_{++}^{n}$. Define

$$
x^{r}=\left\|y^{r-1}\right\|^{-1} y^{r-1}, \quad y^{r}=A x^{r}, r \geqslant 1 .
$$

Let

$$
\bar{\lambda}_{r}=\max _{1 \leqslant i \leqslant n} \frac{y_{i}^{r}}{x_{i}^{r}} \quad \text { and } \quad \underline{\lambda}_{r}=\min _{1 \leqslant i \leqslant n} \frac{y_{i}^{r}}{x_{i}^{r}}
$$

We then have

$$
\underline{\lambda}_{0} \leqslant \underline{\lambda}_{1} \leqslant \cdots \leqslant r(A) \leqslant \cdots \leqslant \bar{\lambda}_{1} \leqslant \bar{\lambda}_{0} .
$$

Theorem 2.8

If $A$ is primitive, then both the sequences $\left(x^{r}, \underline{\lambda}_{r}\right)$ and $\left(x^{r}, \bar{\lambda}_{r}\right)$, produced by the power method, converge to $\left(x_{0}, r(A)\right)$, where $x_{0}$ is the positive eigenvector corresponding to the eigenvalue $r(A)$. 


\section{THE $H$-SPECTRAL THEORY FOR NONNEGATIVE TENSORS}

To those readers, who are not familiar with tensors, we recommend [28], in which the basic definitions and properties of tensors are introduced.

In 2005, Qi [2] and Lim [1] independently introduced the notion of eigenvalue problems for tensors. Here, we follow the definition given in [2,29]: Let $\mathcal{A}=\left(a_{i_{1} \cdots i_{m}}\right) \in \mathbb{R}^{[m, n]}$. Given an $n$-vector $x=\left(x_{1}, \cdots, x_{n}\right)$, real or complex, we define the $n$-vector:

$$
\left(\mathcal{A} x^{m-1}\right)_{i}:=\left(\sum_{i_{2}, \ldots, i_{m}=1}^{n} a_{i i_{2} \cdots i_{m}} x_{i_{2}} \cdots x_{i_{m}}\right)_{1 \leqslant i \leqslant n}
$$

and the $n$-vector $x^{[m-1]}:=\left(x_{1}^{m-1}, \ldots, x_{n}^{m-1}\right)$.

Definition 3.1. A pair $(\lambda, x) \in \mathbb{C} \times\left(\mathbb{C}^{n} \backslash\{\boldsymbol{0}\}\right)$ is called an eigenvalue and an eigenvector of $\mathcal{A} \in \mathbb{R}^{[m, n]}$ if they satisfy $\mathcal{A} x^{m-1}=\lambda x^{[m-1]}$. Furthermore, we say $\lambda$ is an $H$-eigenvalue with the corresponding $H$-eigenvector $x$ (or $(\lambda, x)$ is an $H$-eigenpair) of $\mathcal{A}$ if they are both real.

By definition, the eigenvalues only depends on the $n$ homogeneous polynomials $\left(\mathcal{A} x^{m-1}\right)_{i}$, $i=1, \cdots n$. Thus, without loss of generality, one may assume for any fixed $i \in\{1, \cdots, n\}$, the $(m-1)$-order $n$-dimensional tensor $\left(a_{i, i_{2}, \cdots i_{m}}\right)$ is symmetric.

In [2], Qi proved the following conclusions on the eigenvalues of an $m$-order $n$-dimensional symmetric tensor $\mathcal{A}$ :

Theorem 3.2 ( $c f$. Theorems 1 and 6 [2])

1. A number $\lambda \in \mathbb{C}$ is an eigenvalue of $\mathcal{A}$ if and only if it is a root of the characteristic polynomial $\phi(\lambda)=\operatorname{det}(\mathcal{A}-\lambda \mathcal{I})$, where $\mathcal{I}=\left(\delta_{i_{1} \cdots i_{m}}\right)$ denotes the identity tensor, that is,

$$
\delta_{i_{1} \cdots i_{m}}= \begin{cases}1 & \text { if } i_{1}=i_{2}=\cdots=i_{m} \\ 0 & \text { otherwise }\end{cases}
$$

is the Kronnecker symbol.

2. The number of eigenvalues of $\mathcal{A}$ is $d=n(m-1)^{n-1}$. Their product is equal to $\operatorname{det}(\mathcal{A})$, the resultant of $\mathcal{A} x^{m-1}=0$.

3. The sum of all the eigenvalues of $A$ is

$$
(m-1)^{n-1} \operatorname{tr}(\mathcal{A})
$$

where $\operatorname{tr}(\mathcal{A})$ denotes the sum of the diagonal elements of $\mathcal{A}$.

4. If $m$ is even, then $\mathcal{A}$ always has $H$-eigenvalues. $\mathcal{A}$ is positive definite (positive semidefinite) if and only if all of its $H$-eigenvalues are positive (nonnegative).

5. The eigenvalues of $\mathcal{A}$ lie in the following $n$ disks:

$$
\left|\lambda-a_{i, i, \cdots, i}\right| \leqslant \sum\left\{\left|a_{i, i_{2}, \cdots, i_{m}}\right|: 1 \leqslant i_{2}, \cdots, i_{m} \leqslant n,\left\{i_{2}, \cdots, i_{m}\right\} \neq\{i, \cdots, i\}\right\},
$$

for $i=1, \cdots, n$.

Remarks 1. The notion of symmetric tensor is referred to Definition 4.10 later. In fact, the symmetric assumption on $\mathcal{A}$ in [2] in this statement is superfluous. Corresponding to the $n$ homogenous polynomials $\left(\mathcal{A} x^{m-1}\right)_{1}, \cdots,\left(\mathcal{A} x^{m-1}\right)_{n}$ in $n$ variables $x=\left(x_{1}, \cdots, x_{n}\right)$, one defines the determinant to be the resultant of these polynomials:

$$
\operatorname{det}(\mathcal{A})=\operatorname{Res}\left(\left(\mathcal{A} x^{m-1}\right)_{1}, \cdots\left(\mathcal{A} x^{m-1}\right)_{n}\right),
$$

then the characteristic polynomial becomes

$$
\phi(\lambda)=\operatorname{det}(\mathcal{A}-\lambda \mathcal{I})
$$


All the conclusions of the earlier theorem continue to hold without changing the proofs.

In a more general framework, Canny [30] defined the generalized characteristic polynomial, $C(\lambda)$, of a system of homogeneous polynomials $f_{1}, \ldots, f_{n}$ in the variables $x_{1}, \ldots, x_{n}$ to be the resultant of $\left\{f_{1}-\lambda x_{1}^{d_{1}}, \ldots, f_{n}-\lambda x_{n}^{d_{n}}\right\}$, where each $f_{i}$ has total homogeneous degree $d_{i}$.

A symmetric even $m$ order tensor $\mathcal{A}=\left(a_{i_{1} \cdots i_{m}}\right)$ is called positive definite (or positive semidefinite), if

$$
\Sigma_{i_{1}, \cdots, i_{m}=1}^{n} a_{i_{1} \cdots i_{m}} x_{i_{1}} \cdots x_{i_{m}} \geqslant \alpha \sum_{i=1}^{n} x_{i}^{m}, \alpha>0,(\alpha \geqslant 0) \quad \forall x \in \mathbb{R}^{n} .
$$

Definition 3.3. Let $\mathcal{A} \in \mathbb{R}^{[m, n]}$. We call the set $\sigma(\mathcal{A})=\{\lambda \mid \lambda$ is an eigenvalue of $\mathcal{A}\}$ the spectrum of $\mathcal{A}$. The spectral radius $\rho(\mathcal{A})$ of $\mathcal{A}$ is defined to be

$$
\rho(\mathcal{A})=\sup \{|\lambda| \mid \lambda \in \sigma(A)\} .
$$

According to the remark following Theorem 3.2, for any $\mathcal{A} \in \mathbb{R}^{[m, n]}, \sigma(\mathcal{A}) \neq \emptyset$ is a finite set. Hence, the supremum used in the definition of $\rho(\mathcal{A})$ can be replaced by the maximum.

It should be noted that, unlike the spectral radius $r(A)$ for a square matrix $A$, the spectral radius $\rho(\mathcal{A})$ thus defined not only depends on the tensor $\mathcal{A}$ but also on the definition of the eigenvalue (Definition 3.1) of $\mathcal{A}$.

In contrast, the existence of $H$-eigenpairs for a general tensor $\mathcal{A} \in \mathbb{R}^{[m, n]}$ is significantly more challenging; we refer the interested readers to [31-33] for more detailed discussions on that front.

In [1], Lim first proposed to extend the Perron-Frobenius theorems to nonnegative tensors in this setting. He also extended the notion of irreducibility to higher order tensors as follows.

Definition 3.4. A tensor $\mathcal{A}=\left(a_{i_{1} \cdots i_{m}}\right) \in \mathbb{R}^{[m, n]}$ is called reducible, if there exists a nonempty proper index subset $I \subset\{1, \ldots, n\}$ such that

$$
a_{i_{1} \cdots i_{m}}=0, \quad \forall i_{1} \in I, \quad \forall i_{2}, \ldots, i_{m} \notin I .
$$

If $\mathcal{A}$ is not reducible, then we call $\mathcal{A}$ irreducible.

Due to the nonlinear nature of the eigenvalue problem (Definition 3.1) for a higher order tensor, many standard methods used in linear algebra cannot be applied directly. Surprisingly, for the family of nonnegative tensors, the main component of its spectral theory, for example, the generalized Perron-Frobenius theorems and some related results, can be extended completely.

In 2008, Chang et al. gave the first complete proof of the following generalized Perron-Frobenius theorems:

Theorem 3.5 (cf. Theorem 1.3 [32])

If $\mathcal{A} \in \mathbb{R}_{+}^{[m, n]}$, then there exist $\lambda_{0} \geqslant 0$ and a nonnegative vector $x_{0} \neq 0$ such that

$$
\mathcal{A} x_{0}^{m-1}=\lambda_{0} x_{0}^{[m-1]} .
$$

The proof of Theorem 3.5 is based on Brouwer fixed point theorem. As a consequence, it directly asserts the existence of a real eigenvalue of $\mathcal{A} \in \mathbb{R}_{+}^{[m, n]}$, bypassing the resultant theory over the complex field.

Theorem 3.6 (cf. Theorem 1.4 [32])

If $\mathcal{A} \in \mathbb{R}_{+}^{[m, n]}$ is irreducible, then the pair $\left(\lambda_{0}, x_{0}\right)$ in $(H)$ satisfies the following:

1. $\lambda_{0}>0$ is an eigenvalue.

2. $x_{0}>0$, that is, all components of $x_{0}$ are positive.

3. If $\lambda$ is an eigenvalue with nonnegative eigenvector, then $\lambda=\lambda_{0}$. Moreover, the nonnegative eigenvector is unique up to a multiplicative constant.

4. If $\lambda$ is an eigenvalue of $\mathcal{A}$, then $|\lambda| \leqslant \lambda_{0}$. 
The irreducibility condition not only implies the nonnegative eigenvector is unique but it must be positive as well.

From Theorem 3.6, we see that if $\mathcal{A} \in \mathbb{R}_{+}^{[m, n]}$ is irreducible, then $\rho(\mathcal{A})=\lambda_{0}$ is an eigenvalue. Based on Theorem 3.5, the spectral radius of a nonnegative tensor is defined in [34] (which of course coincides with the more general definition ). By the simple approximation $\rho(\mathcal{A}+\epsilon \mathcal{E}) \geqslant \rho(\mathcal{A})$, where $\mathcal{E}$ denotes the unit tensor whose entries are all equal to 1 , it follows that $\rho(\mathcal{A}+\epsilon \mathcal{E}) \rightarrow \rho(\mathcal{A})$ as $\epsilon \rightarrow 0$. Because $\mathcal{A}+\epsilon \mathcal{E}$ is a positive tensor (hence irreducible), passing to the limit, Theorem 3.6 implies that $\rho(\mathcal{A})$ is an eigenvalue even if $\mathcal{A}$ is reducible. Namely,

Proposition 3.7 (cf. Theorem $2.3[34]$ ). For any $\mathcal{A} \in \mathbb{R}_{+}^{[m, n]}$, the spectral radius $\rho(\mathcal{A})$ is an eigenvalue of $\mathcal{A}$.

Analogous to Theorem 2.4, Yang and Yang [34] also proved

Theorem 3.8 (cf. Theorem 3.1 [34])

Let $\mathcal{A} \in \mathbb{R}_{+}^{[m, n]}$ be irreducible. If $\mathcal{A}$ has $k$ distinct eigenvalues of modulus $\rho(A)$, then the eigenvalues are $\rho(A) e^{i 2 \pi j / k}$, where $j=0,1, \cdots, k-1$.

We continue to call this number $k$ the cyclic index of $\mathcal{A}$.

Similar to the Collatz-Wielandt Theorem 2.7, Chang et al. extended the minimax characterization of the spectral radius $\rho(\mathcal{A})$ in [32] as follows:

Theorem 3.9 (cf. Theorem 4.2 [32])

Assume $\mathcal{A} \in \mathbb{R}_{+}^{[m, n]}$ is irreducible, then

$$
\min _{x \in \mathbb{R}_{++}^{n}} \max _{\left\{i \mid x_{i}>0\right\}} \frac{\left(\mathcal{A} x^{m-1}\right)_{i}}{x_{i}^{m-1}}=\rho(\mathcal{A})=\max _{x \in \mathbb{R}_{++}^{n}} \min _{\left\{i \mid x_{i}>0\right\}} \frac{\left(\mathcal{A} x^{m-1}\right)_{i}}{x_{i}^{m-1}} .
$$

Inspired by Theorem 3.9, $\mathrm{Ng}$ et al. [35] proposed the following algorithm for calculating the spectral radius:

1. Choose $x^{0} \in \mathbb{R}_{++}^{n}$. Let $y^{0}=\mathcal{A}\left(x^{(0)}\right)^{m-1}$ and set $k:=0$.

2. Compute

$$
\begin{aligned}
x^{(k+1)} & =\frac{\left(y^{(k)}\right)^{\left[\frac{1}{m-1}\right]}}{\left\|\left(y^{(k)}\right)^{\left[\frac{1}{m-1}\right]}\right\|}, \\
y^{(k+1)} & =\mathcal{A}\left(x^{(k+1)}\right)^{m-1}, \\
\underline{\lambda}_{k+1} & =\min _{1 \leqslant i \leqslant n} \frac{\left(y^{(k+1)}\right)_{i}}{\left(x_{i}^{(k+1)}\right)^{m-1}}, \\
\bar{\lambda}_{k+1} & =\max _{1 \leqslant i \leqslant n} \frac{\left(y^{(k+1)}\right)_{i}}{\left(x_{i}^{(k+1)}\right)^{m-1}} .
\end{aligned}
$$

The iteration stops whenever $\underline{\lambda}_{k}=\bar{\lambda}_{k}$, which yields the largest eigenvalue $\lambda_{0}$ and the associated eigenvector. 
If the iteration does not terminate in finite time, are the sequences $\left\{\underline{\lambda}_{k}, x^{(k)}\right\}\left\{\bar{\lambda}_{k}, x^{(k)}\right\}$ convergent? In order to give a complete and satisfactory answer to this question, one needs certain condition resembles primitivity for matrices.

By defining the nonlinear map $\mathcal{T}_{\mathcal{A}}(x):=\left(A x^{m-1}\right)^{\left[\frac{1}{m-1}\right]}$ on $\mathbb{R}_{+}^{n}$ associated with the tensor $\mathcal{A}$, Chang et al. [36] enabled the composition of the tensor $\mathcal{A}$ with itself and extended the definition of primitivity to tensors.

Definition 3.10. An irreducible nonnegative tensor $\mathcal{A}$ is said to be primitive if the only nonempty subset of the boundary of $\mathbb{R}_{+}^{n}$, which is invariant under $\mathcal{T}_{\mathcal{A}}$ is $\{\mathbf{0}\}$.

Based on the notion of primitive tensors, they proved

Theorem 3.11 (cf. Theorem 4.5 [36])

If $\mathcal{A} \in \mathbb{R}_{+}^{[m, n]}$ is primitive, then its cyclic index is 1 .

Theorem 3.12 (cf. Theorems 2.7, 2.11 [36])

Let $\mathcal{A} \in \mathbb{R}_{+}^{[m, n]}$, then the following statements are equivalent:

1. $\mathcal{A}$ is primitive.

2. $\exists r \in \mathbb{N}$ such that $\mathcal{T}_{\mathcal{A}}^{r}\left(\mathbb{R}_{+}^{n} \backslash\{\mathbf{0}\}\right) \subset \mathbb{R}_{++}^{n}$, that is, $\mathcal{T}_{\mathcal{A}}^{r}$ is strongly positive.

3. $\exists r \in \mathbb{N}$ such that $\mathcal{T}_{\mathcal{A}}^{r}$ is strictly increasing.

Theorem 3.13 (cf. Theorem 5.3 [36])

If $\mathcal{A} \in \mathbb{R}_{+}^{[m, n]}$ is primitive, let $\left\{\underline{\lambda}_{k}, \bar{\lambda}_{k} ; x^{(k)}\right\}$ be as defined in Theorem 3.9, then $\underline{\lambda}_{k}, \bar{\lambda}_{k} \rightarrow \rho(\mathcal{A})$ and $x^{(k)} \rightarrow x^{*}$, that is, $x^{(k)}$ converges to the eigenvector with respect to $\rho(\mathcal{A})$.

Moreover, Chang et al. proved

Theorem 3.14 (cf. Theorem 5.7 [36])

Let $\mathcal{A} \in \mathbb{R}_{+}^{[m, n]}$ be irreducible. Both the sequences $\left\{\underline{\lambda}_{k}\right\}$ and $\left\{\bar{\lambda}_{k}\right\}$, which are defined in Theorem 3.9, converge to $\rho(\mathcal{A})$ for an arbitrary initial value $x^{0} \in \mathbb{R}_{+}^{n} \backslash\{\boldsymbol{0}\}$ if and only if $\mathcal{A}$ is primitive.

From the definition, it is easily seen

Corollary 3.15 (cf. Corollary 3.8 [36]). Let $\mathcal{A} \geqslant 0$ be irreducible. Then, $\mathcal{A}+\alpha \mathcal{I}$ is primitive, where $\mathcal{I}$ is the identity tensor and $\alpha>0$.

Corollary 3.16 (cf. Corollary 3.7 [36]). If $\mathcal{A} \geqslant 0$ is essentially positive (i.e., $\mathcal{T}_{\mathcal{A}}$ is strongly positive), then $\mathcal{A}$ is primitive.

Combining these results, the convergence results in Zhang and Qi [37] and Yang et al. [38] follow readily. According to [38,39], one may modify the algorithm proposed by $\mathrm{Ng}$ et al. to any irreducible nonnegative tensor $\mathcal{A}$ by adding $\alpha \mathcal{I}$ to $\mathcal{A}$, then $\rho(\mathcal{A})$ can be obtained by subtracting $\alpha$ in the end.

Zhang et al. [40] proved the linear convergence of the earlier algorithm for calculating $\rho(\mathcal{A})$.

Comparing the earlier results for tensors to the classical Perron-Frobenius theorems for nonnegative matrices, we note the similarities as well as the differences between the two settings: similar to nonnegative matrices, the existence of a nonnegative eigenvector with nonnegative eigenvalue for any nonnegative tensor has been established. Furthermore, under Lim's irreducibility definition, the eigenvalue is positive, unique among eigenvalues with nonnegative eigenvectors, and the largest in modulus.

However, unlike matrices, such $\lambda_{0}$ may not be geometrically simple in general. 
Definition 3.17 (cf. Definition 3.1 [32]). Let $\lambda$ be an eigenvalue of $\mathcal{A}$. We say $\lambda$ has real geometric multiplicity $q$, if the maximum number of linearly independent real eigenvectors corresponding to $\lambda$ equals $q$. If $q=1$, then $\lambda$ is called real geometrically simple.

The following example was given in [32].

Example 3.18. Let $\mathcal{A}=\left(a_{i j k}\right) \in \mathbb{R}_{+}^{[3,2]}$ be such that $a_{111}=a_{222}=1, a_{122}=a_{211}=\epsilon$ for $0<\epsilon<1$, and $a_{i j k}=0$ for other $(i j k)$. Then, the eigenvalue problem becomes

$$
\left\{\begin{array}{l}
x_{1}^{2}+\epsilon x_{2}^{2}=\lambda x_{1}^{2} \\
\epsilon x_{1}^{2}+x_{2}^{2}=\lambda x_{2}^{2} .
\end{array}\right.
$$

We have $\lambda_{0}=1+\varepsilon$, with eigenvectors $u_{1}=(1,1)$ and $u_{2}=(1,-1)$. Hence, the real geometric multiplicity of $\lambda_{0}=1+\varepsilon$ is 2 .

With this in mind, we seek sufficient conditions on $\mathcal{A} \in \mathbb{R}_{+}^{[m, n]}$ to ensure the geometric simplicity of $\rho(\mathcal{A})$.

Chang in [41] studied the generalized Perron-Frobenius theorem in Banach space and introduced the notion of semistrong positivity for a positively 1-homogeneous, strictly increasing, compact, and continuous operator. This will lead to the geometric simplicity for this kind of operators.

We now review some basic terminologies.

Definition 3.19. Let $X$ be a real Banach space, which has a positive cone $P$ with nonempty interior $\operatorname{int}(P)$. We write $x \geqslant 0$ if $x \in P ; x>0$ if $x \in \operatorname{int}(P)$ A mapping $T: X \rightarrow X$ is said to be nonnegative if $0 \leqslant x$ implies $0 \leqslant T x$; it is said to be strictly positive if $0<x$ implies $0<T x$; it is said to be strongly positive if $0 \leqslant x$ and $x \neq 0$ imply $0<T x$.

Definition 3.20. A mapping $T: X \rightarrow X$ is said to be increasing if $x \leqslant y$ implies $T x \leqslant T y$; it is said to be strictly increasing, if further, $x<y$ implies $T x<T y$; it is said to be strongly increasing if $x \leqslant y$ and $x \neq y$ imply $T x<T y$.

Definition 3.21. Let $P$ be a positive cone in a real Banach space $X$, and $\mathcal{T}: X \rightarrow X$ be a continuous map $\mathcal{T}: P \rightarrow P$. Assume $\operatorname{int}(P) \neq \emptyset$. $\mathcal{T}$ is called semistrongly positive, if $\forall x \in \dot{P} \backslash \operatorname{int}(P)$, there exists $x^{*} \in P^{*}$ such that

$$
\left\langle x^{*}, \mathcal{T} x\right\rangle>0=\left\langle x^{*}, x\right\rangle .
$$

where $\dot{P}=P \backslash\{0\}$, and $\langle\cdot, \cdot\rangle$ is the duality between $X^{*}$ and $X$.

The proof of the following theorem, which offers an alternative proof of Theorem 3.6 and yields the geometric simplicity result, can be found in [41] Theorem 4.8:

Theorem 3.22

Let $\mathcal{T}$ be a semistrongly positive, increasing, 1-homogeneous, compact, and continuous mapping, and

$$
r^{*}(\mathcal{T})=\inf _{x \in \dot{P}} \sup _{x^{*} \in P^{*}(x)} \frac{\left\langle x^{*}, \mathcal{T} x\right\rangle}{\left\langle x^{*}, x\right\rangle}>0,
$$

where $P^{*}(x)=\left\{x^{*} \in P^{*} \mid\left\langle x^{*}, x\right\rangle>0\right\}$. Then, $\lambda_{0}=r^{*}(\mathcal{T})$ is the unique positive eigenvalue with nonnegative eigenvector $x_{0}$. In fact, the eigenvector $x_{0} \in \operatorname{int}(P)$.

If $\lambda$ is a real eigenvalue of $\mathcal{T}$, then $|\lambda| \leqslant \lambda_{0}$.

If further, $\exists r \in N$, such that $T^{r}$ is strictly increasing, then the eigenvalue $\lambda_{0}$ is geometrically simple.

Now, we return to eigenvalues for tensors.

The proof of Lemma 4.6 in [41] also leads to the following.

Lemma 3.23. A nonnegative tensor $\mathcal{A}$ is irreducible if and only if the operator $\mathcal{T}_{\mathcal{A}}$ is semistrongly positive. 
When $m$ is even, the tensor $\mathcal{A} \in \mathbb{R}_{+}^{[m, n]}$ defines a nonlinear operator on $\mathbb{R}^{n}$ :

$$
\mathcal{T}_{\mathcal{A}}(x)=\left(\mathcal{A} x^{m-1}\right)^{\frac{1}{m-1}},
$$

which maps $\mathbb{R}_{+}^{n}$ to $\mathbb{R}_{+}^{n}$ and is 1-homogeneous.

Concerning the geometric simplicity of $\rho(\mathcal{A})$, without loss of generality, we may assume that $\mathcal{A}$ is primitive. In this case, $\mathcal{T}_{\mathcal{A}}: \mathbb{R}^{n} \rightarrow \mathbb{R}^{n}$, which maps $\mathbb{R}_{+}^{n}$ to $\mathbb{R}_{+}^{n}$, is an increasing, 1-homogeneous, semistrongly positive, compact, and continuous mapping. According to Theorem 3.12, $\exists r \in \mathbb{N}$, such that $\mathcal{T}_{\mathcal{A}}^{r}$ is strictly increasing.

Observing that when $m$ is even, $m-1$ is odd, so

$$
\mathcal{A} x^{m-1}=\lambda x^{m-1} \Leftrightarrow \mathcal{T}_{\mathcal{A}}(x)=\lambda^{\frac{1}{m-1}} x, \quad \forall x \in \mathbb{R}^{n} .
$$

Theorem 3.22 now implies

Theorem 3.24 (cf. Theorem 3.1 [42])

Let $\mathcal{A} \in \mathbb{R}_{+}^{[m, n]}$. If $\mathcal{A}$ is irreducible and $m$ is even, then $\rho(\mathcal{A})$ is real geometrically simple.

It is evident the results of Yang-Yang [42] and Pearson [43] (for essential positive tensors) on the geometric simplicity of the largest eigenvalue can be derived from Theorem 3.24.

The notion of irreducibility for nonnegative $n \times n$ matrices $A=\left(a_{i j}\right)$ has many equivalent forms. Among which, two popular definitions are commonly adopted throughout the existing literature. One of them is given by Definition 2.2. This definition of reducibility/irreducibility of a square matrix $A$ focuses on whether or not $A$ has nontrivial invariant coordinate subspaces, which is a combinatorially based. The other equivalent definition of irreducibility is given by the directed graph $G(A)$ associated to $A$, (i.e., $V=\{1,2 \cdots, n\}$ and an directed edge $(i, j) \in E(A)$ if $\left.a_{i j}>0\right)$ ). $A$ is irreducible if and only if $G(A)$ is strongly connected, that is, for any ordered pair of nodes $i$ and $j$, there exists a directed path connecting $i$ to $j$.

When one extends the notion of irreducibility to nonnegative higher order tensors, these two approaches may lead to different consequences. Following the first approach, we have the definition of irreducibility as given in Definition 3.4.

In 2009, following the graph theory based approach, Friedland et al. [44] introduced the notion of weakly irreducible tensors. It is defined as follows.

Given a nonnegative tensor $\mathcal{A}=\left(a_{i_{1} \cdots i_{m}}\right) \in \mathbb{R}_{+}^{[m, n]}$, it is associated to a directed graph $G(\mathcal{A})=(V, E(\mathcal{A}))$, where $V=\{1,2, \cdots, n\}$ and a directed edge $(i, j) \in E(\mathcal{A})$ if there exists indices $\left\{i_{2}, \cdots, i_{m}\right\}$ such that $j \in\left\{i_{2}, \cdots, i_{m}\right\}$ and $a_{i i_{2} \cdots i_{m}}>0$, that is,

$$
\Sigma_{j \in\left\{i_{2}, \cdots, i_{m}\right\}} a_{i i_{2}, \cdots i_{m}}>0 .
$$

Definition 3.25. A nonnegative tensor $\mathcal{A} \in \mathbb{R}_{+}^{[m, n]}$ is called weakly irreducible if the associate directed graph $G(\mathcal{A})$ is strongly connected.

It is equivalent to say (see $\mathrm{Hu}$ in [45]) that the matrix $M(\mathcal{A})=\left(m_{i j}\right)$ is irreducible, where

$$
m_{i j}=\sum_{j \in\left\{i_{2}, \cdots, i_{m}\right\}} a_{i i_{2}, \cdots i_{m}} .
$$

It is straightforward to show an irreducible nonnegative tensor is weakly irreducible, but the following example is a weakly irreducible tensor, which is reducible according to Definition 3.4.

Example 3.26 (cf. [46]). Let $\mathcal{A} \in \mathbb{R}_{+}^{[4,3]}$ be given by

$$
a_{1111}=a_{1123}=a_{2223}=a_{3113}=1 \quad \text { and } \quad a_{i j k l}=0 \quad \text { elsewhere. }
$$

In [44], Friedland et al. discovered that a series of results obtained by Nussbaum [47, 48], Burbanks et al. [49], Gaubert and Gunawardena [50], and so on. on order preserving mappings as well as 
on positively 1-homogeneous monotone functions can be applied to the nonnegative tensors setting. Applying these results, they reproved Theorem 3.6 under the weakly irreducible condition:

Theorem 3.27

Assume that $\mathcal{A} \in \mathbb{R}_{+}^{[m, n]}$ is weakly irreducible. Then, there exists a unique positive $H$-eigenvector with positive eigenvalue.

It is imperative to distinguish the results of Theorems 3.27 and 3.6 because the weak irreducibility assumption is weaker than irreducibility, Theorem 3.27 establishes the existence of a positive $H$-eigenpair under a weaker assumption. However, Theorem 3.6 asserts the positive $H$-eigenvector is unique up to a nonnegative multiplier in $\mathbb{R}_{+}^{n} \backslash\{\mathbf{0}\}$, and there are no $H$-eigenvectors on the boundary of the positive cone. Whereas, Theorem 3.27 asserts that the positive $H$-eigenvector is unique in $\mathbb{R}_{++}^{n}$ but does not rule out the possibility of an $H$-eigenvector on the boundary of $\mathbb{R}_{+}^{n}$. From a topology point of view, there are two essentially different existence proofs for the classical PerronFrobenius theorem for nonnegative matrices and its generalizations to monotone operators in the literature: one approach is based on Brouwer fixed point theorem. This idea can be traced back to Alexandroff and Hopf in 1935. In 1940, Rutman continued in this vein to reprove Jentsch's theorem on integral equations, which is an infinite dimensional analog of the Perron-Frobenius theorem, by using Schauder fixed point theorem. The other approach is to reduce the eigenvalue problem to a contraction mapping by using the Hilbert projective metric. This idea can be traced back to Samuelson in 1956, Birkhoff in 1957, Thompson in 1963 and Nussbaum [48]. The proof of Theorem 3.6 follows Brouwer fixed point theorem, and the proof of Theorem 3.27 follows the approach of the contraction mapping.

Friedland et al. [44] also proved the convergence of the power algorithm proposed by $\mathrm{Ng}$ et al. continues to hold for a weakly primitive nonnegative tensor (i.e., in the definition of a primitive tensor, the irreducibility condition is replaced by weak irreducibility).

While many important properties of $\rho(\mathcal{A})$ are still enjoyed by the nonnegative weakly irreducible tensors, some others may be lost nevertheless under this weaker notion. In a recent paper [46], Yang and Yang showed using the Example 3.26,

$$
x_{1}=(-0.410215,0.231207,0.33885) \quad \text { and } \quad x_{2}=(5.03736,2.83918,4.16102)
$$

are both approximated eigenvectors corresponding to $\rho(\mathcal{A}) \approx 1.46557$. So, $\rho(\mathcal{A})$ of a nonnegative even order weakly irreducible is not necessarily real geometrically simple, contrasting to the conclusion of Theorem 3.24.

In addition to the results mentioned earlier, there are various extensions on the positiveness of the tensors, for example, Zhang and Qi [37] studied the weakly positive tensors; Hu et al. [51] studied the strictly nonnegative tensors. Further developments can be found in [38-40, 52-56, 86, 87]. Extensions to rectangular nonnegative tensors, essentially nonnegative tensors, copositive tensors, completely positive tensors, and $M$-tensors can be found in [52,54,57-64].

\section{THE $Z$-SPECTRAL THEORY FOR NONNEGATIVE TENSORS}

Parallel to the eigenvalue (resp. $H$-eigenvalue) problem of $\mathcal{A} \in \mathbb{R}^{[m, n]}$, there are other types of eigenvalue problems of $\mathcal{A}$, for example, [1,2,29,31]. In particular, we will investigate the following:

Definition 4.1 (cf. [29]). Let $\mathcal{A} \in \mathbb{R}^{[m, n]}$. A pair $(\lambda, x) \in \mathbb{C} \times\left(\mathbb{C}^{n} \backslash\{\mathbf{0}\}\right)$ is called an $E$-eigenvalue and $E$-eigenvector (or simply E-eigenpair) of $\mathcal{A}$ if they satisfy the equation

$$
\begin{cases}\mathcal{A} x^{m-1} & =\lambda x, \\ x^{\top} x & =1 .\end{cases}
$$

We call $(\lambda, x)$ a $Z$-eigenpair if they are both real.

Independently, Lim also defined eigenvalues for tensors in [1]. Lim defined eigenvalues for general real tensors in the real field. The $\ell^{2}$-eigenvalues of tensors defined by Lim are $Z$-eigenvalues 
of Qi [2], while the $\ell^{m}$-eigenvalues of tensors defined by Lim are $H$-eigenvalues in Qi [2]. Notably, Lim proposed a multilinear generalization of the Perron-Frobenius theorem based upon the notion of $\ell^{m}$-eigenvalues ( $H$-eigenvalues) of tensors.

When $m$ is even, the $E$-characteristic polynomial of $\mathcal{A}$ is defined to be the resultant

$$
\psi_{\mathcal{A}}(\lambda)=\operatorname{Res}_{x}\left(\mathcal{A} x^{m-1}-\lambda\left(x^{\top} x\right)^{\frac{m-2}{2}} x\right) .
$$

It is a polynomial of $\lambda$.

When $m$ is odd, it is defined in [29] to be the resultant:

$$
\psi_{\mathcal{A}}(\lambda)=\operatorname{Res}_{x, x_{0}}\left(\mathcal{A} x^{m-1}-\lambda x_{0}^{m-2} x, x^{\top} x-x_{0}^{2}\right) .
$$

We say that $\mathcal{A}$ is regular if the following system has no nonzero complex solutions:

$$
\left\{\begin{aligned}
\mathcal{A} x^{m-1} & =0 \\
x^{\top} x & =0
\end{aligned}\right.
$$

For a regular tensor, for all $m$, the $E$-characteristic polynomial reads as

$$
\psi_{\mathcal{A}}(\lambda)=\operatorname{Res}_{x, x_{0}}\left(\mathcal{A} x^{m-1}-\lambda x_{0}^{m-2} x, x^{\top} x-x_{0}^{2}\right) .
$$

In [2], Qi also established the following conclusions on the $E$-eigenvalues of an $m$-order $n$-dimensional symmetric tensor $\mathcal{A}$ :

Theorem 4.2 (cf. Theorems 1-4 [29]) 1 . When $\mathcal{A}$ is regular, a complex number is an $E$-eigenvalue of $\mathcal{A}$ if and only if it is a root of its $E$-characteristic polynomial.

2. The $Z$-eigenvalues always exist. An even order symmetric tensor is positive definite if and only if all of its $Z$-eigenvalues are positive.

3. If $\mathcal{A}$ and $\mathcal{B}$ are orthogonally similar, then they have the same $E$-eigenvalues and $Z$ eigenvalues.

4. If $\lambda$ is the $Z$-eigenvalue of $\mathcal{A}$ with the largest absolute value and $x$ is a $Z$-eigenvector associated with it, then $\lambda x^{m}$ is the best rank-one approximation of $\mathcal{A}$, that is,

$$
\left\|\mathcal{A}-\lambda x^{m}\right\|_{F}=\sqrt{\|\mathcal{A}\|_{F}^{2}-\lambda^{2}}=\min \left\{\left\|\mathcal{A}-\alpha u^{m}\right\|_{F}: \alpha \in \mathbb{R}, u \in \mathbb{R}^{n},\|u\|_{2}=1\right\},
$$

where $\|\cdot\|_{F}$ is the Frobenius norm.

Theorem 4.2 (4) indicates that $Z$-eigenvalues play an important role in the best rank-one approximation. The best rank-one approximation of higher order tensors has extensive engineering and statistical applications, such as Statistical Data Analysis [65-67].

Both of the eigenvalue problem and the $E$-eigenvalue problem for tensors are nonlinear in nature with various new and important applications in numerical multilinear algebra, image processing, higher order Markov chains, spectral hypergraph theory, the study of quantum entanglement, and so on. However, their chief difference lies in that the eigenvalue problem is equivalent to finding nontrivial solutions of a system of homogeneous multivariate polynomial equations of the same degree, whereas the $E$-eigenvalue problem is equivalent to finding nontrivial solutions of a system of inhomogeneous multivariate polynomial equations.

It was shown in a recent paper by $\mathrm{Li}$ et al. [20] that the $E$-characteristic polynomial of a given tensor $\mathcal{A} \in \mathbb{R}^{[m, n]}$ (not necessarily symmetric) is in fact invariant under the action of the orthogonal group.

As a consequence, all $E / Z$-eigenvalues of $\mathcal{A}$ are orthogonal invariants of $\mathcal{A}$. The significance of the orthogonal invariance of the $E / Z$-eigenvalues indicates many results on the $Z$-eigenvalues of nonnegative tensors will remain valid for a broader class of tensors, which are not necessarily nonnegative themselves but are orthogonally similar to nonnegative tensors. Unfortunately, the $H$-eigenvalues are not orthogonally invariant. 
Ni et al. [68] showed that for even $m>2$, the degree of the $E$-characteristic polynomial $\psi_{\mathcal{A}}(\lambda)$ of a generic tensor $\mathcal{A}$ is

$$
d_{E}=\frac{(m-1)^{n}-1}{m-2}=(m-1)^{n-1}+(m-1)^{n-2}+\cdots+(m-1)+1 .
$$

They conjectured that the assumption on $m$ being even is not necessary. Later, Cartwright and Sturmfels proved the conjecture in [19]. Furthermore, Cartwright and Sturmfels observed (cf. Proposition 3.4 [19]) the following implications of the relationship between the $E$-eigenvalues and the $E$-characteristic polynomial $\psi_{\mathcal{A}}(\lambda)$ as follows:

1. The set of all $E$-eigenvalues of $\mathcal{A}$ consists of all complex numbers, which implies.

2. The set of all $E$-eigenvalues of $\mathcal{A}$ is infinite, which implies.

3 . The $E$-characteristic polynomial $\psi_{\mathcal{A}}(\lambda)$ vanishes identically.

In the $E$-eigenvalue problem, the regular assumption for a tensor is very important, this can be seen in the following example [19]:

Example 4.3. Let $\mathcal{A}=\left(a_{i j k}\right) \in \mathbb{C}^{[3,2]}$, where

$$
a_{111}=a_{221}=1, \quad a_{112}=a_{222}=i, \quad a_{i j k}=0 \text { otherwise. }
$$

We solve the system

$$
\begin{cases}x_{1}^{2}+i x_{1} x_{2} & =\lambda x_{1} \\ x_{1} x_{2}+i x_{2}^{2} & =\lambda x_{2}\end{cases}
$$

It is easily seen that all $\lambda \neq 0$ are $E$-eigenvalues of $\mathcal{A}$.

The striking difference between the eigenvalues of $\mathcal{A}$ and the $E$-eigenvalues of $\mathcal{A}$ is depicted by the following result because of Cartwright and Sturmfels:

Theorem 4.4 (cf. Proposition 3.3 [19])

The set of $E$-eigenvalues of $\mathcal{A}$ is either a finite set or it consists of all complex numbers in the complement of a finite set.

The following versions of the generalized Perron-Frobenius theorems for the $Z$-eigenvalues as well as other types of eigenvalues of a nonnegative tensor $\mathcal{A}$ were established in Chang et al. [32,69].

\section{Theorem 4.5}

If $\mathcal{A} \in \mathbb{R}_{+}^{[m, n]}$, then there exists a $Z$-eigenvalue $\lambda_{0} \geqslant 0$ and a nonnegative $Z$-eigenvector $x_{0} \neq 0$ of $\mathcal{A}$ such that $\mathcal{A} x_{0}^{m-1}=\lambda_{0} x_{0}$.

Theorem 4.6

If $\mathcal{A} \in \mathbb{R}_{+}^{[m, n]}$ is irreducible, then the pair $\left(\lambda_{0}, x_{0}\right)$ in Theorem 4.5 satisfy

1. The eigenvalue $\lambda_{0}$ is positive.

2. The eigenvector $x_{0}$ is positive, that is, all components of $x_{0}$ are positive.

However, unlike the $H$-eigenpairs for a nonnegative irreducible tensor $\mathcal{A}$, neither the positive $Z$-eigenvalue nor the associated positive $Z$-eigenvector of $\mathcal{A}$ has to be unique in general, see Errata [32] and Example 2.7 in [69].

Similar to the notion of spectrum set and spectral radius for a tensor $\mathcal{A} \in \mathbb{R}^{[m, n]}$, Chang et al. in [69] introduced

Definition 4.7. Let $\mathcal{A} \in \mathbb{R}^{[m, n]}$. We define the $Z$-spectrum of $\mathcal{A}$ to be

$$
\mathcal{Z}(\mathcal{A})=\{\lambda \in \mathbb{R} \mid \lambda \text { is a } Z \text {-eigenvalues of } \mathcal{A}\} .
$$


Assume $\mathcal{Z}(\mathcal{A}) \neq \emptyset, \varrho(\mathcal{A}):=\sup \{|\lambda| \mid \lambda \in \mathcal{Z}(\mathcal{A})\}$ is called the $Z$-spectral radius of $\mathcal{A}$.

According to Theorem 4.5, for a nonnegative tensor $\mathcal{A} \in \mathbb{R}_{+}^{[m, n]}, \mathcal{Z}(\mathcal{A}) \neq \emptyset$, so the $\mathcal{Z}$-spectral radius $\varrho(\mathcal{A})$ is well defined. But, different from the $H$-spectral radius $\rho(\mathcal{A}), \varrho(\mathcal{A})$ may not be itself a positive $Z$-eigenvalue of $\mathcal{A}$, see Example 3.3 in [69].

Based on the earlier observations, we introduce the following.

Definition 4.8 (cf. [69]). Let $\mathcal{A} \in \mathbb{R}_{+}^{[m, n]}$. We define the nonnegative spectrum of $\mathcal{A}$,

$$
\Lambda(\mathcal{A})=\left\{\lambda \geqslant 0 \mid \exists x \in \mathbb{R}_{+}^{n} \cap S^{n-1} \text { satisfying } \mathcal{A} x^{m-1}=\lambda x\right\},
$$

where $S^{n-1}$ is the standard unit sphere in $\mathbb{R}^{n}$.

It is important to notice the set $\Lambda(\mathcal{A})$ is not necessarily a finite set in general as shown by Example 3.5 [69].

Proposition 4.9 (cf. Proposition 3.6 [69]). Let $\mathcal{A} \in \mathbb{R}_{+}^{[m, n]}$. Then, $\Lambda(\mathcal{A}) \neq \emptyset$ is a compact subset.

For $\mathcal{A} \in \mathbb{R}_{+}^{[m, n]}$, we define

$$
\lambda^{*}=\max \{\lambda \in \Lambda(\mathcal{A})\} .
$$

Besides the family of nonnegative tensors, the family of (weakly) symmetric tensors constitutes another special yet important object to study. We recall the following from [2,31].

Definition 4.10. $\mathcal{A} \in \mathbb{R}^{[m, n]}$ is called symmetric if

$$
a_{i_{1} \cdots i_{m}}=a_{\sigma\left(i_{1} \cdots i_{m}\right)} \quad \text { for all } \sigma \in \widetilde{S}_{m},
$$

where $\mathfrak{S}_{m}$ denotes the permutation group of $m$ indices.

It was first called the 'super-symmetric' tensor but was suggested changing to be 'symmetric tensor' by [70]. In that paper, it also demonstrates equivalence to the usual coordinate-free definition of a symmetric tensor in algebra.

The notion of weakly symmetric tensors is introduced in [31].

Definition 4.11. $\mathcal{A} \in \mathbb{R}^{[m, n]}$ is called weakly symmetric if the associated homogeneous polynomial

$$
\mathcal{A} x^{m}=f_{\mathcal{A}}(x):=\sum_{i_{1}, i_{2}, \ldots, i_{m}=1}^{n} a_{i_{1} i_{2} \cdots i_{m}} x_{i_{1}} x_{i_{2}} \cdots x_{i_{m}}
$$

satisfies $\nabla f_{\mathcal{A}}(x)=m \mathcal{A} x^{m-1}$.

It is also shown in [31] that a symmetric tensor is necessarily weakly symmetric, but the converse is not true in general. Furthermore, if $\mathcal{A} \in \mathbb{R}^{[m, n]}$ is weakly symmetric, by homogeneity, we find

$$
\mathcal{A} x^{m}=f_{\mathcal{A}}(x)=\frac{1}{m}\left\langle\nabla f_{\mathcal{A}}(x), x\right\rangle=\left\langle\mathcal{A} x^{m-1}, x\right\rangle,
$$

where $\langle\cdot, \cdot\rangle$ denotes the standard inner product on $\mathbb{R}^{n}$. We define

$$
\bar{\lambda}:=\max _{x \in S^{n-1}} f_{\mathcal{A}}(x)=\max _{x \in S^{n-1}} \mathcal{A} x^{m}
$$

and we have

Theorem 4.12 (cf. Theorem 3.11 [69])

Assume $\mathcal{A} \in \mathbb{R}_{+}^{[m, n]}$ is weakly symmetric. Then,

$$
\bar{\lambda}=\lambda^{*}=\varrho(\mathcal{A})
$$


Definition 4.13 (cf. [69]). Let $\mathcal{A} \in \mathbb{R}_{+}^{[m, n]}$. We say $\mathcal{A}$ is non-degenerate if for all $x \in \mathbb{R}_{+}^{n} \backslash\{\mathbf{0}\}$, $\left(\mathcal{A} x^{m-1}\right)_{i}$ and $x_{i}$ do not vanish simultaneously for all $i \in\{1, \cdots, n\}$.

It is straightforward to show if $\mathcal{A}$ is irreducible, then $\mathcal{A}$ is non-degenerate, see Section 4 [69] for detail. However, the converse is not true in general, for example, Example 4.7 [69].

Definition 4.14 (cf. [69]). Let $\mathcal{A} \in \mathbb{R}_{+}^{[m, n]}$ be non-degenerate. We define the following two functions for all $x \in \mathbb{R}_{+}^{n} \backslash\{\mathbf{0}\}$ :

$$
\nu_{*}(x):=\min _{1 \leqslant i \leqslant n} \frac{\left(\mathcal{A} x^{m-1}\right)_{i}}{x_{i}} \text { and } \quad v^{*}(x):=\max _{1 \leqslant i \leqslant n} \frac{\left(\mathcal{A} x^{m-1}\right)_{i}}{x_{i}} .
$$

In the definition, it may happen $v^{*}(x)=\infty$.

Definition 4.15 (cf. [69]). We define

$$
\varrho_{*}:=\sup _{x \in \mathbb{R}_{++}^{n} \cap S^{n-1}} v_{*}(x) \text { and } \varrho^{*}:=\inf _{x \in \mathbb{R}_{++}^{n} \cap S^{n-1}} \nu^{*}(x) .
$$

We then have a similar max-min characterization of $\varrho(\mathcal{A})$ :

Theorem 4.16 (cf. Theorem 4.5 [69])

Assume $\mathcal{A} \in \mathbb{R}_{+}^{[m, n]}$ is weakly symmetric and irreducible. Then,

1. $\Lambda(\mathcal{A})$ is contained in the closed interval $\left[\varrho^{*}, \varrho_{*}\right]$, that is, $\Lambda(\mathcal{A}) \subseteq\left[\varrho^{*}, \varrho_{*}\right]$.

2. $\varrho(\mathcal{A})=\bar{\lambda}=\lambda^{*}=\varrho_{*}$.

In order to compute $\varrho(\mathcal{A})$, we adapt an iterative algorithm known as the shifted symmetric higher order power method, proposed by Kolda and Mayo [71] when $m$ is even. Although the algorithm as well as its convergence analysis are given under the assumption $\mathcal{A} \in \mathbb{R}^{[m, n]}$ being symmetric, the entire process nonetheless continues to work successfully when we only assume $\mathcal{A}$ is weakly symmetric. We refer the interested reader to [71] for a more in-depth discussion on this subject.

We now adapt the shifted symmetric higher order power method (cf. Algorithm 2 [71]) as follows. Given a weakly symmetric tensor $\mathcal{A} \in \mathbb{R}_{+}^{[m, n]}$.

Step 0. Choose $x_{(0)} \in \mathbb{R}_{+}^{n} \backslash\{\mathbf{0}\}$, set $\lambda_{0}=\mathcal{A} x_{(0)}^{m}$, and choose the shift constant

$$
\alpha=\left\lceil m \sum_{i_{1}, \cdots, i_{m}=1}^{n} a_{i_{1} \ldots i_{m}}\right\rceil,
$$

where $\lceil\gamma\rceil$ is the ceiling function, that is, it equals the smallest integer no less than $\gamma$.

Set $k:=0$.

Step 1. Set $y_{(k+1)}:=\mathcal{A} x_{(k)}^{m-1}+\alpha x_{(k)}$.

Step 2. Compute

$$
\begin{aligned}
x_{(k+1)} & :=\frac{y_{(k+1)}}{\left\|y_{(k+1)}\right\|} \\
\lambda_{k+1} & :=\mathcal{A} x_{(k+1)}^{m} .
\end{aligned}
$$

Because $\alpha$ is large enough, the function $f_{\mathcal{A}}(x)+\alpha\|x\|^{m}$ becomes convex on $\mathbb{R}^{n}$. For even $m$, the convergence of the sequence $\lambda_{k}$ is guaranteed. 


\section{HIGHER ORDER MARKOV CHAINS}

Eigenvalue theory for nonnegative matrices plays an important role in the study of finite Markov chain. A finite Markov chain is a stochastic process $X_{0}, X_{1}, \cdots$, with values in $\{1, \ldots, n\}$. Let

$$
\operatorname{Prob}\left(X_{t+1}=i \mid X_{t}=j\right)=p_{i j} .
$$

We call $P=\left(p_{i j}\right)$ the transition probability matrix; clearly, $p_{i j} \geqslant 0$, and

$$
\sum_{i=1}^{n} p_{i j}=1 \quad \forall j=1, \cdots, n .
$$

A nonnegative matrix satisfying the earlier conditions is called a stochastic matrix.

Let $\xi_{t} \in \mathbb{R}^{n}$ be the distribution of $X_{t}$, that is,

$$
\left(\xi_{t}\right)_{i}=\operatorname{Prob}\left(X_{t}=i\right), \text { and } \sum_{i=1}^{n}\left(\xi_{t}\right)_{i}=1,
$$

then we have

$$
\xi_{t+1}=P \xi_{t} \text { and } \sum_{i=1}^{n}\left(\xi_{t+1}\right)_{i}=1 .
$$

If $\xi \in \mathbb{R}^{n}$ is the eigenvector of $P$, with $\xi \geqslant 0$ and $\sum_{i=1}^{n} \xi_{i}=1$, then $P \xi=\xi$. In this sense, $\xi$ corresponds to an invariant distribution of the Markov chain.

In case $P$ is irreducible, according to the classical Perron-Frobenius theorem, $\xi$ corresponds to the largest eigenvalue 1 , which is simple. One can show

$$
\xi_{t} \rightarrow \xi, \text { as } t \rightarrow \infty
$$

In this sense, $\xi$ is also called the equilibrium distribution.

Motivated by the demand of multirelational data mining, $\mathrm{Ng}$ et al. discovered intriguing new connections of the $Z$-eigenvalue problem to the transition probability tensors of higher order Markov chains in a series of their recent work $[16,72,73]$. They proposed a framework (HAR) that can be used to compute hub, authority, and relevance scores in multirelational data query. The basic facts of higher order Markov chains can be found in [74].

A higher order Markov chain is an extension of the finite Markov chain, in which the stochastic process $X_{0}, X_{1}, \cdots$ with values in $\{1,2, \cdots, n\}$ has the transition probabilities:

$$
0 \leqslant p_{i_{1} i_{2} \cdots i_{m}}=\operatorname{Prob}\left(X_{t}=i_{1} \mid X_{t-1}=i_{2}, \ldots, X_{t-m+1}=i_{m}\right) \leqslant 1
$$

where

$$
\sum_{i_{1}=1}^{n} p_{i_{1}, i_{2}, \cdots, i_{m}}=1, \quad 1 \leqslant i_{2}, \ldots, i_{m} \leqslant n .
$$

Thus, we have a tensor $\mathcal{P} \in \mathbb{R}_{+}^{[m, n]}$ consisting of $n^{m}$ entries in between 0 and 1:

$$
\mathcal{P}=\left(p_{i_{1} i_{2} \ldots i_{m}}\right), \quad 1 \leqslant i_{1}, i_{2}, \ldots, i_{m} \leqslant n,
$$

satisfying (3). We call it a transition probability tensor.

Let the probability distribution at time $t$ be $\xi^{(t)} \in \Delta_{n}$, where

$$
\Delta_{n}=\left\{x=\left(x_{1}, \cdots, x_{n}\right) \in \mathbb{R}_{+}^{n}: \sum_{j=1}^{n} x_{j}=1\right\} .
$$


Then, we have

$$
\xi^{(t+m)}=\mathcal{P} \xi^{(t+m-1)} \cdots \xi^{(t)}:=\left(\sum_{i_{2} \cdots i_{m}=1}^{n} p_{i, i_{2} \cdots i_{m}} \xi_{i_{2}}^{(t+m-1)} \cdots \xi_{i_{m}}^{(t)}\right)_{i=1}^{n} \in \Delta_{n}
$$

for $t=1,2, \cdots$.

Assume that

$$
\lim _{t \rightarrow \infty} \xi^{(t)}=\xi^{*}
$$

Then, we have $\xi^{*} \in \Delta_{n}$, and we may call $\xi^{*}$ the stationary probability distribution of the higher order Markov chain.

It follows that $\xi^{*} \in \Delta_{n}$ is an eigenvector of $\mathcal{P}$ with eigenvalue 1 , that is,

$$
\mathcal{P} \xi^{m-1}=\xi .
$$

Although the eigenvalue problem (6) is different from the $\mathcal{Z}$-eigenvalue problem for $\mathcal{P}$,

$$
\begin{cases}\mathcal{P} x^{m-1} & =\lambda x \\ x^{\top} x & =1\end{cases}
$$

they share the same eigenvector (with a positive constant multiplier), but they may correspond to different eigenvalues [14].

Consequently, if $\mathcal{P}$ is irreducible, then a positive solution of (6) exists. This is also proved by Li and $\mathrm{Ng}$ [16]. Moreover, they gave sufficient conditions for the uniqueness of the fixed point problem (6) inside the simplex $\Delta_{n}$. Under the same conditions (essentially to ensure $\mathcal{P}$ being a contraction mapping on $\Delta_{n}$ ), $\mathrm{Li}$ and $\mathrm{Ng}$ [16] established linear convergence of the power method

$$
y^{(k+1)}=\mathcal{P}\left(y^{(k)}\right)^{m-1} .
$$

Although it has been noted in the previous section that for an arbitrary irreducible nonnegative tensor the largest $\mathcal{Z}$-eigenvalue may correspond to more than one positive $\mathcal{Z}$-eigenvector (or equivalently, the fixed points of $\mathcal{P}$ inside $\Delta_{n}$ need not be unique for an arbitrary irreducible nonnegative tensor), it was unclear whether every irreducible transition probability tensor has a unique fixed point, until a counter example appeared in [14]:

Example 5.1. Let $\mathcal{P} \in \mathbb{R}_{+}^{[4,2]}$ be the positive (hence irreducible) transition probability tensor defined by

$$
\begin{aligned}
& p_{1111}=0.872, \quad p_{1112}=\frac{2.416}{3}, \quad p_{1121}=\frac{2.416}{3}, \quad p_{1122}=\frac{0.616}{3} \\
& p_{1211}=\frac{2.416}{3}, \quad p_{1212}=\frac{0.616}{3}, \quad p_{1221}=\frac{0616}{3}, \quad p_{1222}=0.072 \\
& p_{2111}=0.128, \quad p_{2112}=\frac{0.584}{3}, \quad p_{2121}=\frac{0.584}{3}, \quad p_{2122}=\frac{2.384}{3} \\
& p_{2211}=\frac{0.584}{3}, \quad p_{2212}=\frac{2.384}{3}, \quad p_{2221}=\frac{2384}{3}, \quad p_{2222}=0.928
\end{aligned}
$$

The fixed points of $\mathcal{P}(6)$ are solutions the following system:

$$
\left\{\begin{array}{l}
\left(\mathcal{P} x^{3}\right)_{1}=0.872 x_{1}^{3}+2.416 x_{1}^{2} x_{2}+0.616 x_{1} x_{2}^{2}+0.072 x_{2}^{3}=x_{1}, \\
\left(\mathcal{P} x^{3}\right)_{2}=0.128 x_{1}^{3}+0.584 x_{1}^{2} x_{2}+2.384 x_{1} x_{2}^{2}+0.928 x_{2}^{3}=x_{2}, \\
x_{1}+x_{2}=1, \quad x_{1}, x_{2} \in[0,1] .
\end{array}\right.
$$

It is easy to verify that both

$$
v_{1}=(0.2,0.8), \quad v_{2}=(0.6,0.4)
$$

satisfy the system. 
Consequently, finding sufficient conditions to ensure the uniqueness of the fixed point for an irreducible transition probability tensor becomes an important task to be carried out. In this direction, we mention the works of Hu and Qi [15] and Chang and Zhang [14].

Another important problem in this aspect is the convergence of the probability distribution $\xi^{(t)}$ as time $t \rightarrow \infty$, that is, (5).

For second-order Markov chain, under some additional conditions, Hu and Qi [15] established the convergence of (5).

\section{APPLICATIONS TO SPECTRAL HYPERGRAPH THEORY}

Spectral graph theory is a well developed area of graph theory with applications to combinatorics, computer science, neural networks, and social sciences, and so on. A graph can be encoded by its adjacency matrix $M$. A main theme in spectral graph theory is to study the properties of the graph and the eigenvalues or singular values of $M$. Lim [1] pointed out that a potential application of the largest eigenvalue of a nonnegative tensor is on hypergraphs. However, to extend the existing results and methodology from spectral graph theory to spectral hypergraph theory, a more sophisticated approach is required, namely via tensors.

We adopt the following standard definition of hypergraphs, for more details, we refer to $[5,7-13,75-78]$.

Definition 6.1. A hypergraph $\mathcal{H}$ is a pair $(V, E)$, where $E \subseteq \mathcal{P}(V)$, the power set of $V$. The elements of $V=V(\mathcal{H})$ are called vertices, and the elements of $E=E(\mathcal{H})$ are called edges. A hypergraph $H$ is said to be $k$-uniform for an integer $k \geqslant 2$ if, for all $e \in E(\mathcal{H})$, the cardinal number of the subset, $|e|=k$. The term $k$-graph is often used in place of $k$-uniform hypergraph.

By definition, a 2-graph is a graph.

Definition 6.2. The adjacency tensor $\mathcal{A}_{\mathcal{H}}$ for a uniform $m$-graph $\mathcal{H}=(V, E)$, denoted $\mathcal{A}_{\mathcal{H}}=$ $\left(a_{i_{1}, \cdots, i_{m}}\right) \in \mathbb{R}^{[m, n]}$, where $n$ is the number of the set $V$, is the symmetric tensor given by

$$
a_{i_{1}, \cdots, i_{m}}=\frac{1}{(m-1) !}\left\{\begin{array}{cc}
1, & \text { if } i_{1}, \cdots i_{m} \in E \\
0, & \text { otherwise }
\end{array}\right.
$$

Characteristic polynomial plays an important role in the spectral graph theory. In the study of the spectrum of hypergraphs, the characteristic polynomial $\phi(\lambda)=\operatorname{det}(\mathcal{A}-\lambda \mathcal{I})$ for the tensor $\mathcal{A}$ is used extensively. Because our subject is the spectral theory for nonnegative tensors, we shall focus on the applications of results obtained in previous sections.

Definition 6.3. $\mathcal{H}$ is said to be $m$-regular if every $v \in V$ is adjacent to exactly $m$ hyper-edges. A hypergraph $\mathcal{H}=(V, E)$ is said to be $k$-partite or $k$-colorable if there exists a partition of the vertices $V=V_{1} \cup \cdots \cup V_{k}$ such that for any $k$ vertices $i_{1}, \cdots, i_{k}$ with $a_{i_{1} \cdots i_{k}} \neq 0, i_{1}, \cdots, i_{k}$ must each lie in a distinct $V_{i}$ for $1 \leqslant i \leqslant k$.

Let $\lambda_{\max }$ be the $\mathcal{H}$-eigenvalue (in the sense of Definition 3.1) of a $m$-graph $\mathcal{H}$ with largest modulus. It follows from Theorem 3.6 that

Theorem 6.4 (cf. Theorem 3.7 [5])

For any nonempty $m$-graph $\mathcal{H}, \lambda_{\max }$ can be chosen to be a positive real number. If $\mathcal{H}$ is connected, then a corresponding eigenvector $\mathbf{x}$ can be chosen to be strictly positive.

This result is subsequently generalized by Pearson and Zhang to multigraphs using a different approach.

Theorem 6.5 (cf. Theorem 1 [12])

Let $\mathcal{H}$ be a connected $m$-multigraph on $n$ vertices, then 
1. There exists $\left(\lambda_{0}, x_{0}\right) \in \mathbb{R}_{++} \times \mathbb{R}_{++}^{n}$ an $H$-eigenpair of $\mathcal{A}_{\mathcal{H}}$, where

$$
\lambda_{0}=\rho\left(\mathcal{A}_{\mathcal{H}}\right)=\max _{x \in S_{+}} \mathcal{A}_{\mathcal{H}} x^{m}
$$

is the $H$-spectral radius of $\mathcal{A}_{\mathcal{H}}$, where $S_{+}=\left\{x \in \mathbb{R}_{+}^{n} \mid \sum_{i=1}^{n} x_{i}^{m}=1\right\}$.

2. The positive eigenvector $x_{0}$ is unique (up to a positive multiplier) in $\mathbb{R}_{++}^{n}$,

see also [79].

Further discussions on the $H$ as well as $Z$-eigenvalues/eigenvectors of the adjacency tensor can be found in [5] and [12].

Recently, there has been rapid developments in the discovery of spectral properties of the Laplacian and signless Laplacian of uniform hypergraphs. According to Qi et al. [8], we have the following:

Definition 6.6. For a $k$-uniform hypergraph $\mathcal{H}=(V, E)$ on $n$ vertices, let $\mathcal{D}$ be a $k$-th order $n$ dimensional diagonal tensor with its diagonal element $d_{i \ldots i}$ being $d_{i}$, the degree of vertex $i$, for all $i \in[n]$. Then, $\mathcal{L}:=\mathcal{D}-\mathcal{A}_{\mathcal{H}}$ is the Laplacian tensor of the hypergraph $\mathcal{H}$ and $\mathcal{Q}:=\mathcal{D}+\mathcal{A}_{\mathcal{H}}$ is the signless Laplacian tensor of the hypergraph $\mathcal{H}$.

Both the Laplacian tensor and the signless Laplacian tensor of the hypergraph $\mathcal{H}$ have exhibited promising spectral properties analogous to the Laplacian matrix for graphs. For a more in depth exposition, we refer the interested readers to $[8,9,78]$.

For a hypergraph $\mathcal{H}$, a function $f: V(\mathcal{H}) \rightarrow[r]$ is a (weak) proper $r$-coloring of $\mathcal{H}$ if for every edge $e=\left\{v_{1}, v_{2}, \ldots, v_{k}\right\}$, there exist $i \neq j$ such that $f\left(v_{i}\right) \neq f\left(v_{j}\right)$. The (weak) chromatic number of $\mathcal{H}$, denoted $\chi(\mathcal{H})$, is the minimum $r$ such that $\mathcal{H}$ has a proper $r$-coloring. In the same paper, Cooper and Dutle showed

Theorem 6.7 (cf. Theorem 3.9 [5])

For any $k$-graph $H, \chi(H) \leqslant \lambda_{\max }+1$.

Given an undirected graph $G$ and a positive integer $k \leqslant \omega(G)$, the clique number, that is, the maximum size of a clique (a subset of mutually adjacent vertices in $\mathrm{V}$ ) in $G$, one can build a hypergraph $H$, which is called the $k$-clique $(k+1)$-graph of $G$ having $k$-cliques of $G$ as vertices and $(k+1)$-cliques of $G$ as edges.

By using the spectral radius of the hypergraph $H$, Bulo and Pelillo $[3,4]$ obtained new upper and lower bounds for $\omega(G)$ :

Theorem 6.8 (cf. Theorem 5 [3])

Let $G$ be an undirected graph with clique number $\omega(G)$ and $H$ be a $k$-clique $(k+1)$-graph of $G$ with spectral radius $r(H)$. Then,

$$
\omega(G) \leqslant \frac{r(H)}{k !}+k
$$

Theorem 6.9 (cf. Theorem 6 [3])

Let $G$ be an undirected graph with clique number $\omega(G)$ and $H$ a $k$-clique $(k+1)$-graph of $G$ with spectral radius $r(H)$ and Perron eigenvector $x_{0}$. Then,

$$
\omega(G) \geqslant \psi^{-1}\left(\frac{r(H)}{k !\left\|x_{0}\right\|_{k}^{k+1}}\right),
$$

where

$$
\psi_{k}(x)=(x-k)\left(C_{k}^{n}\right)^{\frac{1}{k}} .
$$


Although the Laplace matrix, which plays an important role in spectral graph theory, is not a nonnegative matrix, after modifications, it can be reduced to a nonnegative matrix. The Laplace matrix for an undirected graph $G$ is symmetric and semipositive definite, the smallest eigenvalue $\mu_{1}(G)$ is 0 , and the multiplicity of 0 is the number of connected components of $G$. Moreover, the second smallest Laplace eigenvalue $\mu_{2}(G)$ is called the algebraic connectivity of the graph $G$, one has $\mu_{2}(G)>0$ if and only if $G$ is connected. The earlier notions and results are extended to $k$-uniform hypergraphs in $[7,77]$, where the $Z$-eigenvalues for tensors are used.

\section{APPLICATIONS TO QUANTUM ENTANGLEMENT}

The quantum entanglement problem is a central problem in quantum information [80]. In 2003, Wei and Goldbart [81] introduced geometrical measure for quantum entanglement. Wei and Goldbart [81] conjectured that the nearest separable state for a symmetric state can be chosen to be symmetric. Hayashi et al. [82] proved the conjecture for symmetric states with nonnegative amplitudes. Hübener et al. [83] proved the conjecture in general. Also see Orús et al. [84]. The computation of the symmetric pure states with nonnegative amplitudes was carried out by Wei and Goldbart [81] for some ground states, and systematically for symmetric pure multipartite qubit states by Chen et al. [85]. Very recently, Hu et al. [22] established the link between the geometric measure of entanglement of pure states with nonnegative amplitudes and the spectral theory of nonnegative tensors.

An $m$-partite pure state $|\Psi\rangle$ of a composite quantum system is an element in a Hilbert tensor product space $\mathcal{H}=\bigotimes_{k=1}^{m} \mathcal{H}_{k}$, with $\langle\Psi \mid \Psi\rangle=1$, where the dimension of $\mathcal{H}_{k}$ is $d_{k}$ for $k=1, \ldots, m$. A separable (Hartree) $m$-partite state $|\Phi\rangle \in \mathcal{H}$ has the form $|\Phi\rangle=\bigotimes_{k=1}^{m}\left|\phi^{(k)}\right\rangle$ with $\left|\phi^{(k)}\right\rangle \in \mathcal{H}_{k}$ and $\|\left|\phi^{(k)}\right\rangle \|=1$ for $k=1, \ldots, m$. A state is called entangled if it is not separable.

For a given $m$-partite pure state $|\Psi\rangle \in \mathcal{H}$, its nearest separable state $|\Phi\rangle=\bigotimes_{k=1}^{m}\left|\phi^{(k)}\right\rangle$ can be described by the maximal overlap:

$$
G(\Psi)=\max _{|\Phi\rangle=\bigotimes_{k=1}^{m}\left|\phi^{(k)}\right\rangle}|\langle\Psi \mid \Phi\rangle| .
$$

The geometric measure is defined as [81]

$$
E_{G}(|\Psi\rangle)=1-G(\Psi)^{2} .
$$

It was shown that the maximal overlap in (8) is equal to the largest entanglement eigenvalue $\lambda$ $[23,81]$ :

$$
\left\{\begin{aligned}
\langle\Psi|\left(\otimes_{j \neq k}\left|\phi^{(j)}\right\rangle\right) & =\lambda\left\langle\phi^{(k)}\right|, \\
\left.\left(\otimes_{j \neq k}\left\langle\phi^{(j)}\right|\right) \Psi\right\rangle & =\lambda\left|\phi^{(k)}\right\rangle, \\
\|\left|\phi^{(k)}\right\rangle \| & =1, k=1, \ldots, m .
\end{aligned}\right.
$$

A state $|\Psi\rangle \in \mathcal{H}=\bigotimes_{k=1}^{m} \mathcal{H}_{k}$ is called nonnegative if there exist orthonormal bases $\left\{\left|e_{i}^{(k)}\right\rangle\right\}_{i=1}^{d_{k}}$ for $\mathcal{H}_{k}$ such that $a_{i_{1} \ldots i_{m}}:=\left\langle\Psi\left|\left(\left|e_{i_{1}}^{(1)}\right\rangle \cdots\left|e_{i_{m}}^{(m)}\right\rangle\right) \geqslant 0\right.\right.$ for all $i_{j}=1, \ldots, d_{j}$ and $j=1, \ldots, m$. The $d_{1} \times \cdots \times d_{m}$ tensor consisting of $a_{i_{1} \ldots i_{m}}$ is denoted by $\mathcal{A}_{\Psi}$. When $\mathcal{H}_{1}=\cdots=\mathcal{H}_{m}, \mathcal{A}_{\Psi}$ is symmetric if and only if $|\Psi\rangle$ is symmetric in the sense of quantum information.

When $|\Psi\rangle$ is symmetric, (8) reduces to [83]

$$
G(\Psi)=\max _{|\Phi\rangle=|\phi\rangle}|\langle\Psi \mid \Phi\rangle| .
$$

The following theorem in [22] established the link between the geometric measure of entanglement of pure states with nonnegative amplitudes and the largest $Z$-eigenvalues of nonnegative tensors. 
Theorem 7.1

If $|\Psi\rangle \in \mathcal{H}$ is symmetric and nonnegative, then

$$
G(\Psi)=\varrho\left(\mathcal{A}_{\Psi}\right) .
$$

Hu et al. [22] further showed that for the geometric measure of entanglement for pure states with nonnegative amplitudes, the nonsymmetric ones can be converted to the symmetric ones, via symmetric embedding [86]. Based on these, the results on $Z$-eigenvalues of nonnegative tensors can be applied to the computation of the geometric measure of entanglement of any pure states with nonnegative amplitudes.

\section{ACKNOWLEDGEMENTS}

We are thankful to the four referees for their valuable comments, which helped us to improve our paper greatly. The second author's work was supported by the Hong Kong Research Grant Council (Grant No. PolyU 501909, 502510, 502111 and 501212). The first author's work was supported by the Chinese NSF No. 61121002 .

\section{REFERENCES}

1. Lim L. Singular values and eigenvalues of tensors: A variational approach. Proceedings of 1st IEEE International Workshop on Computational Advances of Multi-Tensor Adaptive Processing (CAMSAP), Puerto Vallarta, December 13-15, 2005; 129-132.

2. Qi L. Eigenvalues of a real supersymmetric tensor. Journal of Symbolic Computation 2005; 40:1302-1324.

3. Bulò SR, Pelillo M. New bounds on the clique number of graphs based on spectral hypergraph theory. In Learning and Intelligent Optimization. Springer Verlag: Berlin, 2009; 45-48.

4. Bulò SR, Pelillo M. A generalization of the Motzkin-Straus theorem to hypergraphs. Optimization Letters 2009; 3:187-295.

5. Cooper J, Dutle A. Spectra of uniform hypergraphs. Linear Algebra and its Applications 2012; 436:3268-3292.

6. Drineas P, Lim LH. A multilinear spectral theory of hypergraphs and expander hypergraphs 2005.

7. Hu S, Qi L. Algebraic connectivity of an even uniform hypergraph. Journal of Combinatorial Optimization 2012; 24(4):564-579.

8. Hu S, Qi L. The Laplacian of a uniform hypergraph. Journal of Combinatorial Optimization 2013. DOI: 10.1007/s10878-013-9596-x, 2013.

9. Hu S, Qi L. The eigenvectors of the zero Laplacian and signless Laplacian eigenvalues of a uniform hypergraph, 2013. arXiv:1303.4048.

10. Hu S, Qi L, Shao J. Cored hypergraphs, power hypergraphs and their Laplacian eigenvalues. Linear Algebra and Its Applications (in press). arXiv:1304.6839.

11. Hu S, Qi L, Xie J. The largest Laplacian and signless Laplacian H-eigenvalues of a uniform hypergraph, 2013. arXiv:1303.1315.

12. Pearson K, Zhang T. On spectral hypergraph theory of the adjacency tensor, (with Kelly Pearson). Graphs and Combinatorics Published online July 2013. DOI: 10.1007/s00373-013-1340-x.

13. Qi L. $\mathrm{H}^{+}$-eigenvalues of Laplacian and signless Laplacian tensors. Communications in Mathematical Sciences (in press). arXiv:1303.2186.

14. Chang KC, Zhang T. On the uniqueness and nonuniqueness of the $Z$-eigenvector for transition probability tensors. Journal of Mathematical Analysis and Applications 2013; 408(2):525-540. DOI: 10.1016/j.jmaa.2013.04.019.

15. Hu S, Qi L. Convergence of a second order Markov chains, Department of Applied Mathematics, The Hong Kong Polytechnic University, 2011.

16. $\mathrm{Li} \mathrm{W}, \mathrm{Ng} \mathrm{M}$. On the limiting probability distribution of a transition probability tensor. Linear and Multilinear Algebra (in press). DOI: 10.1080/03081087.2013.777436.

17. Qi L, Wang Y, Wu E. D-eigenvalues of diffusion kurtosis tensors. Journal of Computational and Applied Mathematics 2008; 221:150-157.

18. Qi L, Yu G, Wu E. Higher order positive semi-definite diffusion tensor imaging. SIAM Journal on Imaging Sciences 2010; 3:416-433.

19. Cartwright D, Sturmfels B. The number of eigenvalues of a tensor. Linear Algebra and its Applications 2013; 438(2):942-952.

20. Li A, Qi L, Zhang B. E-characteristic polynomials of tensors. Communications in Mathematical Sciences 2013; 11(1):33-53.

21. Balan V, Perminov N. Applications of resultants in the spectral $m$-root framework. Applied Sciences 2010; 12:20-29.

22. Hu S, Qi L, Zhang G. The geometric measure of entanglement of pure states with nonnegative amplitudes and the spectral theory of nonnegative tensors, Department of Applied Mathematics, The Hong Kong Polytechnic University, 2012.

23. Qi L. The minimum Hartree value for the quantum entanglement problem. arXiv: 1202.2983v1. 
24. Zhang F, Zhou B, Peng L. Gradient skewness tensors and local illumination detection for images. Journal of Computational and Applied Mathematics 2013; 237:663-671.

25. Ling C, Nie J, Qi L, Ye Y. Bi-quadratic optimization over unit spheres and semidefinite programming relaxations. SIAM Journal on Optimization 2009; 20:1286-1310.

26. Berman A, Plemmom P. Nonnegative Matrices in the Mathematical Sciences. SIAM: Philadelphia, 1994.

27. Varga R. Matrix Iterative and Its Applications. Springer: Berlin, 1986.

28. Lim LH. Tensors and hypermatrices. In Handbook of Linear Algebra, 2nd Ed. CRC Press, Boca Raton: FL, 2013.

29. Qi L. Eigenvalues and invariants of tensors. Journal of Mathematical Analysis and Applications 2007; 325: 1363-1377.

30. Canny J. Generalized characteristic polynomials. Journal of Symbolic Computation 1990; 9(3):241-250.

31. Chang KC, Pearson K, Zhang T. On eigenvalue problems of real symmetric tensors. Journal of Mathematical Analysis and Applications 2009; 350:416-422.

32. Chang KC, Pearson K, Zhang T. Perron-Frobenius theorem for nonnegative tensors. Communications in Mathematical Sciences 2008; 6(2):5070-520. Errata pp. 1025 (2012).

33. Zhang T. Existence of real eigenvalues of real tensors. Nonlinear Analysis 2011; 74:2862-2868.

34. Yang Q, Yang Y. Further results for Perron-Frobenius Theorem for nonnegative tensors. SIAM Journal on Matrix Analysis and Applications 2010; 31(5):2517-2530.

35. Ng M, Qi L, Zhou G. Finding the largest eigenvalue of a nonnegative tensor. SIAM Journal on Matrix Analysis and Applications 2010; 31(3):1090-1099.

36. Chang KC, Pearson K, Zhang T. Primitivity, the convergence of the NQZ method, and the largest eigenvalue for nonnegative tensors. SIAM Journal on Matrix Analysis and Applications 2011; 32:806-819.

37. Zhang L, Qi L. Linear convergence of an algorithm for computing the largest eigenvalue of a nonnegative tensor. Numerical Linear Algebra with Applications 2012; 19(5):830-841.

38. Yang Y, Yang Q, Li Y. An algorithm to find the spectral radius of nonnegative tensors and its convergence analysis, 2011. arXiv:1102.2668, 14

39. Liu Y, Zhou G, Ibrahim N. An always convergent algorithm for the largest eigenvalue of an irreducible nonnegative tensor. Journal of Computational and Applied Mathematics 2010; 235(1):286-292.

40. Zhang L, Qi L, Xu Y. Linear convergence of the LZI algorithm for weakly positive tensors. Journal of Computational Mathematics 2012; 30:24-33.

41. Chang KC. A nonlinear Krein Rutman theorem. Journal of Systems Science and Complexity 2009; 22:542-554.

42. Yang Q, Yang Y. Geometric simplicity of the spectral radius of nonnegative irreducible tensors. Frontiers of Mathematics in China 2013; 1:129-140.

43. Pearson K. Essentially positive tensors. International Journal of Algebra 2010; 4:421-427.

44. Friedland S, Gaubert S, Han L. Perron-Frobenius theorem for nonnegative multilinear forms and extensions. Linear Algebra and its Applications 2013; 438(2):738-749.

45. Hu S. A note on the positive eigenvector of nonnegative tensors. Optimization Letters 2013; 7(3):519-531.

46. Yang Q, Yang Y. On some properties of nonnegative weakly irreducible tensors, 2011. arXiv:1111.0713, 25.

47. Nussbaum R. Convexity and log convexity for the spectral radius. Linear Algebra and its Applications 1986; 73:59-122.

48. Nussbaum R. Hilbert's projective metric and iterated nonlinear maps. Memoirs of the American Mathematical Society 1988; 75:1-118.

49. Burbanks A, Nussbaum R, Sparrow C. Extensions of order-preserving maps on a cone. Proceedings of the Royal Society of Edinburgh: Section A Mathematics 2003; 133:35-59.

50. Gaubert S, Gunawardena J. The Perron-Frobenius theorem for homogeneous, monotone functions. Transactions of the American Mathematical Society 2004; 356(12):4931-4950.

51. Hu S, Huang Z, Qi L. Strictly nonnegative tensors and nonnegative tensor partition. Science China Mathematics (in press). arXiv: 1111.2138v1 [math.NA], 9 Nov 2011.

52. Qi L. Symmetric nonnegative tensors and copositive Tensors. Linear Algebra and its Applications 2013; 439: 228-238.

53. Yang Q, Zhang L, Zhang T, Zhou G. eds. Spectral theory of nonnegative tensors. Frontiers of Mathematics in China $2013 ; 8: 1-168$.

54. Zhou G, Caccetta L, Qi L. Convergence of an algorithm for the largest singular value of a nonnegative rectangular tensor. Linear Algebra and its Applications 2013; 438(2):959-968.

55. Zhou G, Qi L, Wu SY. Efficient algorithms for computing the largest eigenvalue of a nonnegative tensor. Frontiers of Mathematics in China 2013; 8:155-168.

56. Zhou G, Qi L, Wu SY. On the largest eigenvalue of a symmetric nonnegative tensor. Numerical Linear Algebra with Applications 2013; 20(6):913-928.

57. Chang KC, Qi L, Zhou G. Singular values of a real rectangular tensor. Journal of Mathematical Analysis and Applications 2010; 370:284-294.

58. Chang KC, Zhang T. Multiplicity of singular values for tensors. Communications in Mathematical Sciences 2009; 7:611-625

59. Hu H, Li G, Qi L, Song Y. Finding the maximum eigenvalue of essentially nonnegative symmetric tensors via sum of squares programming on the largest eigenvalue of a symmetric nonnegative tensor. Journal of Optimization Theory and Applications 2013. DOI: 10.1007/s10957-013-0293-9. 
60. Qi L, Xu C, Xu Y. Nonnegative tensor factorization, completely positive tensors and an Hierarchically elimination algorithm, 2013. arXiv: $1305.5344 \mathrm{v} 1$.

61. Yang Q, Yang Y. Singular values of nonnegative rectangular tensors. Frontiers of Mathematics in China 2011; 6:363-378.

62. Zhang L. Linear convergence of an algorithm for the largest singular value of a real rectangular tensor. Frontiers of Mathematics in China 2013; 8:141-153.

63. Zhang L, Qi L, Luo Z, Xu Y. The dominant eigenvalue of an essentially nonnegative tensor. Numerical Linear Algebra with Applications 2013; 20(6):929-941.

64. Zhang L, Qi L, Zhou G. M-tensors and the positive definiteness of a multivariate form, Department of Mathematical Sciences, Tsinghua University, 2012. arXiv:1202.6431v1 [math.NA].

65. De Lathauwer L, De Moor B, Vandewalle J. On the best rank-1 and rank- $\left(R_{1}, R_{2}, \cdots, R_{N}\right)$ approximation of higher-order tensor. SIAM Journal on Matrix Analysis and Applications 2000; 21:1324-1342.

66. Kofidis E, Regalia P. On the best rank-1 approximation of higher-order supersymmetric tensors. SIAM Journal on Matrix Analysis and Applications 2002; 23:863-884.

67. Zhang T, Golub GH. Rank-1 approximation of higher-order tensors. SIAM Journal on Matrix Analysis and Applications 2011; 23:534-550.

68. Ni G, Qi L, Wang F, Wang Y. The degree of the E-characteristic polynomial of an even order tensor. Journal of Mathematical Analysis and Applications 2007; 329:1218-1229.

69. Chang KC, Pearson K, Zhang T. Some variational principles of the $Z$-eigenvalues for nonnegative tensors. Linear Algebra and its Applications 2013; 438:4166-4182.

70. Comon P, Golub G, Lim LH, Mourrain B. Symmetric tensors and symmetric tensor rank. SIAM Journal on Matrix Analysis and Applications 2008; 30:1254-1279.

71. Kolda T, Mayo J. Shifted Power method for computing tensor eigenpairs. SIAM Journal on Matrix Analysis and Applications 2011; 34:1095-1124.

72. Li X, Ng M, Ye Y. MultiRank: co-ranking for objects and relations in multi-relational data, preprint on 14 February 2011. 17th ACM SIGKDD Conference on Knowledge Discovery and Data Mining (KDD-2011), San Diego, CA., $2011 ; 1217-1225$.

73. Li X, Ng M, Ye Y. HAR: hub, authority and relevance scores in multi-relational data for query search. SIAM International Conference on Data Mining, CA., 2012; 141-152.

74. Ching WK, Ng M. Markov Chains: Models, Algorithms and Applications. Springer: New York, NY, 2006.

75. Berge C. Hypergraphs. In North-Holland Mathematical Library 45. North-Holland: Amsterdam, 1989.

76. Cooper J, Dutle A. Computing hypermatrix spectra with Poisson product formula, 2013. arXiv: 1301.4590.

77. Li G, Qi L, Yu G. The $Z$-eigenvalues of a symmetric tensor and its application to spectral hypergraph theory. Numerical Linear Algebra with Applications 2013; 20(6):1001-1029.

78. Xie J, Chang A. H-eigenvalues of the signless Laplacian tensor for an even uniform hypergraph. Frontiers of Mathematics in China 2013; 8:107-127.

79. Lim LH. Foundations of numerical multilinear algebra: decompositions and approximations of tensors. Ph.D. Thesis, 2007. Stanford University, Stanford, CA.

80. Nielsen MA, Chuang IL. Quantum Computing and Quantum Information. Cambridge University Press: Cambridge, 2000.

81. Wei TC, Goldbart PM. Geometric measure of entanglement and applications to bipartite and multipartite quantum states. Physics Review A 2003; 68:042307.

82. Hayashi M, Markham D, Murao M, Owari M, Virmani S. The geometric measure of entanglement for a symmetric pure state with non-negative amplitudes. Journal of Mathematical Physics 2009; 50:122104.

83. Hübener R, Kleinmann M, Wei TC, Guillén CG, Gonzalez-Guillen C, Gühne O. Geometric measure of entanglement for symmetric states. Physics Review A 2009; 80:032324.

84. Orús R, Dusuel S, Vidal J. Equivalence of critical scaling laws for many-body entanglement in the Lipkin-MeshkovGlick Model. Physics Review Letters 2008; 101:025701.

85. Chen L, Xu A, Zhu H. Computation of the geometric measure of entanglement for pure multiqubit states. Physics Review A 2010; 82(3):032301.

86. Yang Q, Yang Y. Further results for Perron-Frobenius Theorem for nonnegative tensors II. SIAM Journal on Matrix Analysis and Applications 2011; 32(4):1236-1250.

87. Zhou G, Caccetta L, Teo KL, Wu SY. Positive polynomial optimization over unit spheres and convex programming relaxations. SIAM Journal on Optimization 2012; 22:987-1008. 